No routine bedside test measures cardiac index directly; the diagnosis of LCOS is currently made based on a number of clinical parameters including lactate, urine output and mixed venous saturations $\left(\mathrm{svO}_{2}\right)$. The efficacy of NIRS has been compared to these parameters in a number of studies.

Averaged cerebral and renal NIRS $<65 \%$ predict a lactate $>3 \mathrm{mmol} / \mathrm{L}$ with a sensitivity of $95 \%$ and a specificity of $83 \%(\mathrm{p}=0.0001$, AUC 0.92).

NIRS can detect the redistribution of blood flow occurring as a physiological response to low cardiac output states, assessed by analysing the difference between somatic (flank) and cerebral NIRS. A NIRS difference between somatic and cerebral NIRS of $10 \%$ is significant $(p<0.001)$.

NIRS also provides a robust indicator of renal dysfunction with an increased risk of doubling the creatinine level every hour the renal NIRS is $<60 \%(\mathrm{p}<0.001)$.

Lower cerebral NIRS during the 60 minutes post bypass is associated with a lower psychomotor development index (PDI) of the Bayley Scales and haemosiderin foci on MRI. Further reports have evaluated the predictive value of NIRS on neurodevelopmental outcomes at two years of age, reporting that receptive communication may also be affected by the NIRS nadir.

Sceptics refer to studies showing that NIRS values can be ascertained from inanimate objects, including root vegetables. This is not surprising since NIRS evaluates light absorption and even vegetables contain substances that can absorb and/or reflect specific wavelengths.

Near-infrared spectroscopy is an exciting development in medical technology which provides the benfit of continuous, real-time, non-invasive monitoring at the bedside. It provides an adjunct to standard investigative modalities, correlating well with transcranial Doppler values and markers of tissue perfusion. NIRS can provide a useful indication that a change in regional blood flow has occurred and interventions performed in response to change in NIRS result in reduced morbidity.

\section{P63 PERIODONTAL HEALTH STATUS IS ASSOCIATED WITH DAILY BLOOD PRESSURE LEVELS IN ADOLESCENTS WITH HYPERTENSION}

Anna Pogodina, Larisa Kolesnikova, Olga Valyavskaya, Lilia Zurbanova, Lyubov Rychkova* Scientific Centre for Family Health and Human Reproduction Problems, Irkutsk, Russian Federation

\subsection{6/archdischild-2019-epa.418}

Aim To establish significant associations between periodontal health status, daily blood pressure (BP) profile and cardiovascular risk factors in adolescents with elevated BP.

Materials and methods A cross-sectional study was conducted. We included 113 adolescents (78 boys) of 10-17 years whose BP level, after repeated office measurements, was at $\geq 95$ th percentile for this age group, height and sex, or $\geq 140 / 90 \mathrm{mmHg}$ in adolescents over 16 years. All adolescents underwent 24-hour blood pressure monitoring, blood tests (lipid profile, fasting glucose, levels of uric acid, ALT and AST), echocardiography (left ventricular mass index, relative wall thickness), anthropometric measurements with BMI calculation, Tanner staging, dental examination (complex dental plaque index, oral hygiene index). We used univariate and multiple regression analyses to evaluate the associations between periodontium state and cardiovascular risk factors (adjusted to sex, age, Tanner stage, BMI, oral hygiene index).

Results Clinical signs of periodontitis were observed in $32.7 \%$ of adolescents. At the same time, no severe forms of periodontitis were diagnosed. Multiple regression analysis showed significant associations of complex dental plaque index with 24-hour systolic, mean and pulse BP levels $(\beta=0.42, p=0.0001 ; \beta=0.31, p=0.003 ; \beta=0.26, p=0.018$ respectively), daytime diastolic BP level $(\beta=0.23, p=0,019)$, and indices of 24-hour systolic BP load $(\beta=0.42, p=0.0001)$ and daytime diastolic BP load $(\beta=0.25, \mathrm{p}=0.006)$. None of other cardiovascular risk factors showed any significant association with periodontium state during the multiple regression analysis.

Conclusion The association between periodontal health status and BP is observed in adolescence, preceding the formation of comorbid associations between periodontitis and lipid and carbohydrate metabolism disorders. Obtained results corroborate examining the periodontal health status and, if necessary, conducting timely preventive activities in adolescents with elevated BP. The results also support evaluation of BP in adolescents with confirmed periodontitis.

\section{P64 PREVALENCE AND CHARACTERISTICS OF CORONARY ARTERITIS IN AWASAKI DISEASE}

Gunay Rasulova*. Azerbaijan Medical University, Baku, Azerbaijan

10.1136/archdischild-2019-epa.419

Prevalence and characteristics of coronary arteritis in Kawasaki disease

Background and aims Kawasaki disease

Kawasaki disease (KD) is an acute, self - limiting systemic vasculitis of unknown aetiology whitch most commonly occurs in children aged 6 month to 5 years, with a peak incidence at 9-11 month.

Kawasaki disease is the most common cause of acquired coronary vessel abnormalities in children.

The aims of this study were to define the frequency and the clinical characteristics of coronary arteritis in Kawasaki disease in Azerbaijan.

Method We included $24 \mathrm{KD}$ patients followed at our hospital from January 2015 to January 2019.

Results Among the 24 patients enrolled 18 had coronary arteritis. The median age of KD patients with coronary arteritis was 28 months. Of the 18 patients developed coronary arteritis 4 patients had aneurysmal lesions and 1 patients had arterial thrombotic occlusions.

Treatment with immunoglobulin and aspirin is effective at reducing the risk of cardiac complications from $25 \%$ to $5 \%$ in the Azerbaijan.

Conclusion The KD patients with coronary arteritis had significantly increased levels of inflamatory markers.Of the 18 patients developed coronary arteritis 2 patients was less than 1 year old and they was the most seriously ill and was aneurysmal lesions.In this patients the coronary arteritis left sequelae and did require additional medications. 